\title{
Opción preferencial por los jóvenes de las clases populares: la experiencia de la Universidad Salesiana de Bolivia $(\mathrm{USB})^{1}$
}

\author{
Willy W. Chambi Zabaleta ${ }^{2}$ \\ Ernesto Murillo Estrada ${ }^{3}$
}

\begin{abstract}
Asegurar una continua reflexión y profundización sobre la identidad y la misión de la presencia salesiana en la educación superior, que se manifiesta en un modelo educativo pastoral universitario y en la opción preferencial por los jóvenes de las clases populares.
\end{abstract}

(Políticas para la Presencia Salesiana en la Educación Superior 2016-2021).

RESUMEN

Un sujeto social en construcción, tanto en su integridad individual y grupal, con grupos de referencia concretos e imaginarios, es centro de las reflexiones políticas, económicas, sociales y educativas de un país, aunque las instituciones en la que éste se desenvuelve hagan poco por él. Hoy en día, los jóvenes representan un gran porcentaje de la población global total. Se estima que hay más de mil millones de jóvenes entre las edades de 15 a 24 años, constituyendo casi un quinto de la población mundial. En nuestro país, Bolivia, un poco más de dos millones se encuentra entre los 12 y 25 años, gran parte de ellos no encuentra una fuente de trabajo y tampoco un centro de estudios. Este grupo social conocido como los "ni-ni” (jóvenes que ni trabajan ni estudian) en Bolivia llegan a los 200 mil, pero, a diferencia de las grandes ciudades, adoptan esta situación no tanto por decisión propia, sino porque no encuentran una oportunidad. Es este panorama, se suma que estos jóvenes no tienen experiencia de trabajo ni formación o entrenamiento. Este contexto político, económico y social, sumado a los cambios de las últimas décadas, influye decisivamente en la conducta y decisiones de los jóvenes de

1 Artículo recibido el 10/9/2018. Artículo aceptado el 30/11/2018.

2 Boliviano, Licenciado en Ciencias de la Educación de la USB. Académico e investigador Universidad Salesiana de Bolivia. E-mail: wchambi@siu.edu

3 Boliviano, Comunicador Social, Filósofo, Sociólogo y Teólogo. Académico e investigador Universidad Salesiana de Bolivia. E-mail: emebol26@hotmail.com 
Opción preferencial por los jóvenes de las clases populares: la experiencia de la universidad salesiana de Bolivia (USB) /Chambi y Murillo

nuestro país. Es en este conjunto de fenómenos interrelacionados que este artículo se propone profundizar.

Palabras clave: Jóvenes, educación, opción preferencial clases populares.

\title{
Preference for young people coming from low social classes: the experience of Universidad Salesiana de Bolivia (USB)
}

\author{
ABSTRACT
}

A social subject under construction, both in its individual and group integrity, with concrete and imaginary reference groups, is at the center of the political, economic, social and educational reflections of a country, although institutions in which it operates do little about. Today, young people represent a large percentage of the total global population. It is estimated that there are more than one billion young people between 15 and 24 years old, constituting almost one fifth of the world's population. In our country, Bolivia, a little more than two million are between 12 and 25 years old, most of them are not able to find a source of work or a center of studies. In Bolivia, this social group known as the "ni-ni" ("neither-nor," young people who neither work nor study) reaches 200,000 , but unlike big cities, they live in this situation not by their own decision, but because they do not find an opportunity. This situation gets worst since these young people have no work experience and lack of training or formation. This political, economic and social context, added to the changes of the last decades, has a decisive influence on the behavior and decisions of young people in our country. This article is aimed at deepening into this set of interrelated phenomena.

Keywords: Young people, education, preference, low social classes

\section{Opção preferencial pelos jovens das classes populares: a experiência da Universidade Salesiana da Bolívia (USB)}

\section{RESUMO}

Um sujeito social em construção, tanto na sua integridade individual e grupal, com grupos de referência concretos e imaginários, é centro das reflexões políticas, econômicas, sociais e educativas de um país, embora as instituições nas que este atua façam pouco por ele. Na atualidade, os jovens representam uma grande porcentagem da população global total. Estimase que haja mais de mil milhões de jovens entre as idades de 15 a 24 anos, constituindo quase um quinto da população mundial. Em nosso país, a Bolívia, um pouco mais de dois milhões encontram-se entre os 12 e 25 anos, grande parte deles não encontra uma fonte de trabalho nem um centro de estudos. Este grupo social conhecido como os "nem-nem" (jovens que nem trabalham nem estudam) na Bolívia chegam aos 200 mil, mas ao contrário 
das grandes cidades, adotam esta situação não simplesmente por decisão própria, mas porque não encontram uma oportunidade. Neste panorama, soma-se o fato de estes jovens não terem experiência de trabalho e a falta de formação ou treinamento. Este contexto político, econômico e social somado às mudanças das últimas décadas influi decisivamente na conduta e decisões dos jovens de nosso país. É este conjunto de fenômenos inter-relacionados, que este artigo se propõe aprofundar.

Palavras-chave: Jovens, educação, opção preferencial, classes populares.

\section{Contexto social de la presencia educativa y pastoral de la USB}

En el ámbito político, Bolivia ha migrado del neoliberalismo, que se había ofertado como única alternativa democrática, a la denominada "Agenda Patriótica 2025" del actual régimen de gobierno a del Sr. Evo Morales. Esta Agenda postula trece pilares para una "Bolivia Digna y Soberana", entre las que se destacan: la erradicación de la pobreza extrema; la socialización y universalización de los servicios básicos con soberanía para Vivir Bien; salud; educación y deporte para la formación de un ser humano integral con identidad propia.

En el ámbito económico, el cierre de muchas fuentes de trabajo formal, el creciente paso al comercio informal, las escasas posibilidades de crecimiento de gran parte de las actividades privadas y la concentración del poder económico en manos de pocos, genera el descontento social. A esto se suma que el Estado se ha convertido en el gran empleador y el acceso a esas fuentes laborales requiere de la adscripción y militancia activa al actual partido de gobierno.

En el ámbito social, el costo del empobrecimiento de los grandes grupos sociales recae sobre las espaldas de muchos jóvenes; se ha afectado los derechos sociales de muchas familias por el difícil acceso a una salud universal y educación de calidad. De acuerdo con Ernesto Pérez de Rada, del Programa de Naciones Unidas para el Desarrollo (PNUD): “Los jóvenes están enfrentando condiciones particularmente difíciles, tanto en lo educativo como lo laboral. Ellos no pueden acceder a un trabajo acorde. El primer empleo 
para los que buscan insertarse no es el mejor, pues acceden a espacios de baja calidad y remuneración" (Pérez de Rada, 2018, s/p).

Por otro lado, en Bolivia, todavía la mayor parte de la extrema pobreza en su dimensión material tiene rostro indígena, campesino, de mujeres, niñas y niños. De lo citado surge la necesidad de contar con una educación competitiva y políticas estatales para capacitarse.

En cuanto a las expectativas de los jóvenes, estudios demuestran que la generación denominada "Millennials" (personas que nacieron a partir de 1998) se inclina por seguir estudios relacionados con la tecnología y muchas universidades tratan de responder esta demanda. Por su parte, la generación Z, estudiantes que actualmente están iniciando sus carreras postsecundarias, demandan un tipo de educación con una fuerte integración tecnológica, flexible y sobre todo autónoma. Este grupo poblacional, por sus características, es más independiente, multiactivo, inmediatista, imaginativo-innovador y autodidacta. Si antes los niños y adolescentes resolvían sus dudas elaborando preguntas para los papás, ahora ellos entran a internet para resolver sus dudas; esta nueva generación es mucho más visual; su buscador en internet ya no es Google, sino YouTube.

En la lectura del discurso político se aspira a que los bolivianos “Tenemos que desarrollar nuestra propia tecnología rompiendo las cadenas de la dependencia. Esta nuestra tecnología tiene un reto estratégico que es el de la industrialización y transformación de nuestros recursos estratégicos para fortalecer vigorosamente nuestra economía" (Agenda Patriótica 2025), pero no se ha avanzado significativamente en este campo.

De acuerdo con el Censo 2012 del Instituto Nacional de Estadísticas de Bolivia (INE), la población boliviana llegó a las 10.027.254 personas, de las cuales 3.128.000 están en la edad anotada. Esta generación de personas jóvenes es mucho más emprendedora, no tiene miedo al cambio y sus círculos de interacción, relacionamiento y discusión están en las redes sociales virtuales. 
Los elementos citados determinan una serie de conductas, como una especie de apoliticismo, que no es otra cuestión que el dolor y la frustración disfrazados de ironía, que se convierte en una indiferencia hacia la identificación o afiliación política de un buen número de personas.

Adicionalmente, la migración juvenil a las grandes ciudades, de acuerdo con el Informe de Desarrollo Humano del Programa de Naciones Unidas para el Desarrollo - 2016 (PNUD), ha ocasionado una sobredemanda de trabajo, obligando a los jóvenes de esta generación a "trabajar de lo que sea".

Los jóvenes tienden a identificarse con los productos globales de consumo, debido a la gran influencia de los medios de comunicación. Los productos globales trazan líneas de pertenencia que rebasan los límites locales (barrio, escuela, universidad) y se establecen otras demarcaciones más universales. De esta manera, internet ejerce una línea de agrupamiento e interacción, especialmente de los jóvenes.

Finalmente, los jóvenes oscilan entre la apropiación de elementos simbólicos, a veces ajenos a ellos, y la resistencia, porque queda la formación de la familia y las costumbres, sumado a los constructos mentales adquiridos en un posible paso por la educación primaria, secundaria y superior.

\section{Propuesta educativa y pastoral de la USB}

La Universidad Salesiana de Bolivia es obra de la Congregación de los Salesianos de Bolivia - Inspectoría Nuestra Señora de Copacabana, reconocida por el Estado Boliviano de acuerdo con las disposiciones de la Resolución Ministerial 068/98 del 2 de marzo 1998 y jerarquizada con Decreto Supremo Nº 1066 de 28 de noviembre de 2011. Su promotor y fundador, R. P. Esteban Bertolusso sdb, italiano de nacimiento y boliviano de corazón, advirtió en la educación superior universitaria una oportunidad para que lo jóvenes de las clases menos favorecidas accedieron a estudios profesionales. 
En la presente gestión, la USB ha cumplido veinte años de vida institucional con vocación educativa y pastoral, atendiendo a una población que supera los ocho mil estudiantes, más de ciento veinte administrativos, casi cuatrocientos docentes de las diferentes ramas del saber humano y con cobertura nacional presente en cinco de las nueve regiones administrativas de Bolivia.

Imagen 1: Cobertura del servicio educativo y pastoral de la USB 2018.

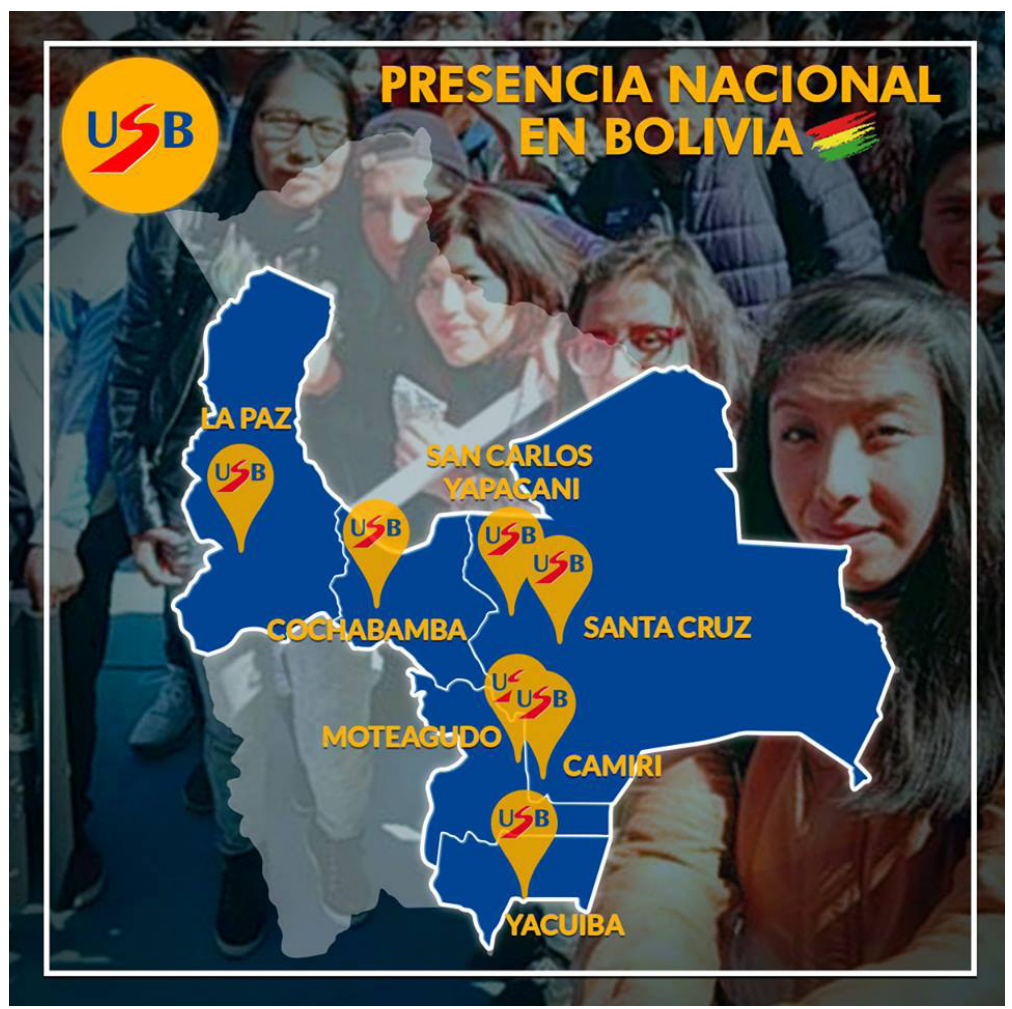

Fuente: Universidad Salesiana de Bolivia, 2018.

Esta presencia a nivel nacional es un reflejo de la intencionalidad institucional de ir en busca de los jóvenes menos favorecidos, ofreciéndoles una propuesta educativa y pastoral alternativa a las tradicionales. La ubicación geográfica de cada una de las subsedes está en plena concordancia con el Artículo 14º de los Reglamentos Generales de los Salesianos de Don Bosco, el cual reza: 
La escuela salesiana sea popular por su ubicación, por la cultura y orientación a las que da la primacía y por lo jóvenes que acoge. Organice servicios útiles a la población de la zona: como cursos de cualificación profesional y cultural, alfabetización y de recuperación, fondos para becas y otras iniciativas semejantes. (Constituciones y Reglamentos, Salesianos de Don Bosco, 2010, p. 139)

La ubicación geográfica de la USB, por tanto, está sometida a este mandato de los Salesianos de Don Bosco. La Sede Central está ubicada en la Zona de Achachicala de la ciudad de La Paz, un barrio periférico y conexo con la ciudad de El Alto, hogar de un buen número de migrantes de las áreas rurales del altiplano boliviano. En Cochabamba, la USB está presente no en la capital del departamento, sino en la ciudad de Colcapirhua, una ciudad próxima a la ciudad capital del departamento. En el departamento de Santa Cruz, la USB está presente en dos municipios: San Carlos-Yapacaní y Camiri, otrora capital petrolera de Bolivia. También está presente en el centro de la ciudad capital, pero solo con servicios de posgrado. En el departamento de Chuquisaca, Monteagudo es la ciudad que acoge a la USB. Finalmente, la presencia de la USB está cercana a la frontera con la República Argentina, en la ciudad de Yacuiba, donde la propuesta de la USB está articulada y en colaboración con la labor diocesana. 
Grafico 1: Estudiantes de la sede central y las subsedes académicas.

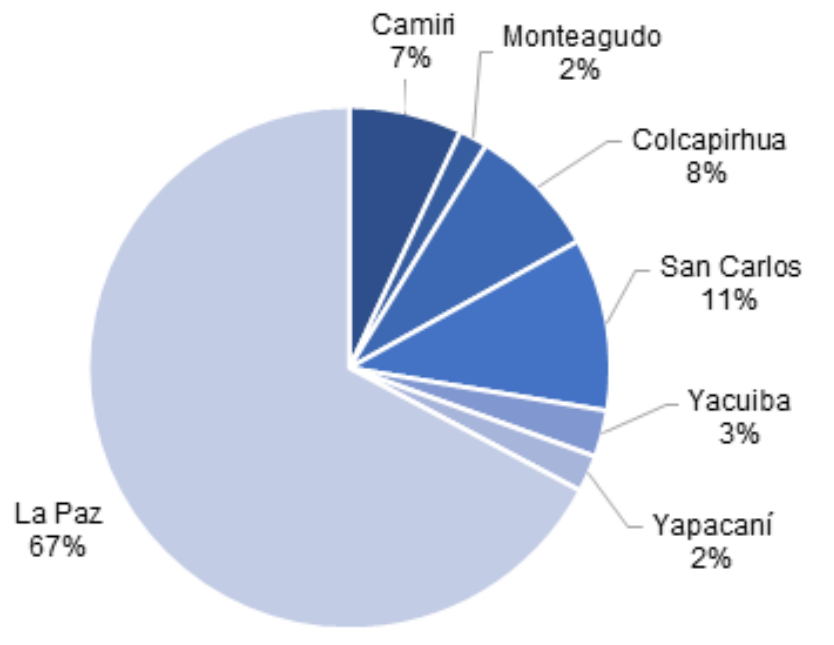

Fuente: Dirección de Planificación, USB. Estudio del Perfil del Estudiante de la USB 2018.

La ubicación geográfica de la USB estaría incompleta sin una articulación con una propuesta educativa y pastoral, alineada a las disposiciones de las constituciones y reglamentos de los salesianos de Don Bosco. De acuerdo con estos documentos, la escuela, los centros profesionales e instituciones de educación superior "...promueven el desarrollo integral del joven mediante la asimilación y la elaboración crítica de la cultura y mediante la educación en la fe, con miras a la transformación cristiana de la sociedad" (Reglamentos y Constituciones, Salesianos de Don Bosco, 2010, p. 138).

Estos preceptos están disgregados en el Modelo Académico de la USB (2016), propuesta cimentada en seis principios institucionales, a saber: (1) pedagogía salesiana, (2) formación integral, (3) atención pastoral y el acompañamiento de cada persona, (4) opción preferencial por lo menos favorecidos y clases populares, (5) promoción del diálogo entre culturas y (6) libertad académica. El punto cuatro, opción preferencial por los menos favorecidos y clases populares, es un principio institucional en el que la USB ha puesto especial atención. En el siguiente apartado, se presentan al- 
gunos gráficos que representan la experiencia de la USB en cuanto a la opción preferencial.

\section{Caracterización del estudiante de la USB}

Es posible afirmar que el perfil del estudiante que estudia en la USB tiene ciertos patrones comunes, aunque no únicos ni homogéneos. En un intento por conocer las características de los jóvenes que frecuentan el servicio educativo y pastoral de la USB, la Dirección de Planificación y Evaluación, luego de haber aplicado cuestionarios para este fin, ha identificado ciertos rasgos que de algún modo describen el "rostro" del estudiante de la Universidad Salesiana de Bolivia.

\section{Los grupos generacionales a los que pertenecen los estudiantes de la USB}

De acuerdo con la categorización del Pew Research Center, son cinco los grupos generacionales presentes en la vida de hoy: (1) Generación silenciosa, aquellos que nacieron entre 1928 y 1945, (2) Baby boomers, nacidos entre 1946 y 1964, (3) Generación X, nacidos entre 1965 y 1980, (4) Generación Y (millenials) nacidos entre 1981 y 1997 y (5) Generación Z, nacidos entre y después de 1998 , cuya característica fundamental es la diversidad y la fuerte utilización de medios y aparatos tecnológicos.

De acuerdo con esta categorización, los resultados de la encuesta señalan que los estudiantes de la USB tienen un matiz bastante interesante. Si bien es cierto que la Universidad es principalmente para los jóvenes, existe la presencia de otras generaciones (ver gráfico 2). 
Gráfico 2: Grupos generacionales de los estudiantes de la USB.

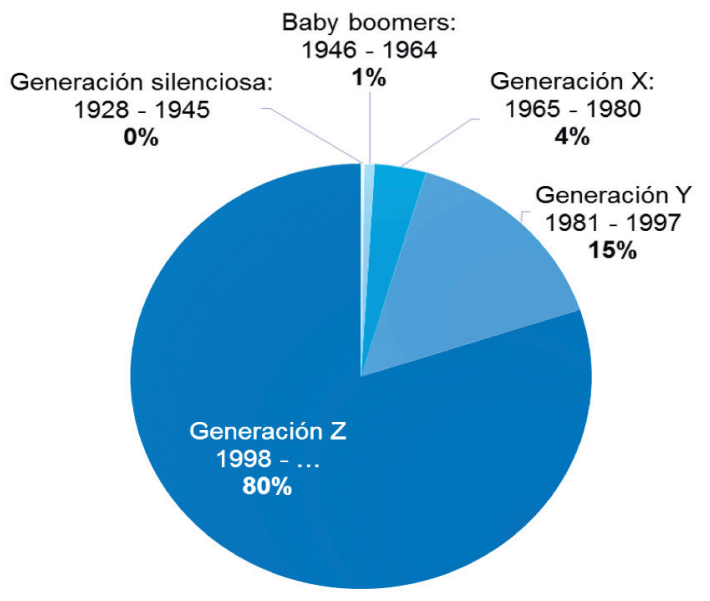

Fuente: Dirección de Planificación, USB. Estudio del Perfil del Estudiante de la USB 2018.

El 80\% de los estudiantes de la USB que iniciaron sus estudios en la gestión 2018 son estudiantes que pertenecen a la Generación Z, es decir, son jóvenes que nacieron en el año 1998 o posterior; es decir, la comunidad estudiantil de la USB tiene rostro joven. Adicionalmente a estos datos, es menester señalar que el 91\% de estos estudiantes son solteros y el $99 \%$ de ellos tiene al menos una cuenta en alguna de las redes sociales, como Facebook, WhatsApp, Instagram, Twitter, Snapchat, entre otros.

Estos resultados coinciden con el reporte Estado TIC presentado por la Agencia de Gobierno Electrónico y Tecnologías de Información y Comunicación (AGETIC), cuyo contenido menciona que el 97\% de los jóvenes de la Generación Z usan Facebook como principal red social, a continuación están WhatsApp y YouTube, con $96 \%$ y $49 \%$ respectivamente.

Indudablemente, este panorama de cambios generacionales vertiginosos nos lleva a cuestionarnos respecto de la pertinencia de nuestra propuesta educativa y pastoral. ¿Cuál es la respuesta de la Universidad ante las expectativas de los "nuevos" jóvenes? ¿Es la 
Universidad una alternativa real para los jóvenes de hoy y sus aspiraciones?

\section{Estudiantes de primera generación}

Es cada vez más común escuchar la expresión "estudiantes de primera generación”. Esta expresión se refiere a “...hijos de padres sin diploma de estudios superiores y sin experiencia en este nivel educacional" (Soto, 2016, p. 1160). Por un lado, esta población estudiantil es particularmente vulnerable, dado que existe mayor probabilidad en la reprobación de asignaturas o, lo que es peor, el abandono de los estudios terciarios; por otro lado, Lahire (1995) advierte que esto podría ser un mito, "el mito de los padres dimitidos". "En efecto, aunque la familia posea un bajo capital cultural, la -educación puede tomar un 'lugar simbólico' en la relación padres-hijos-escuela, contribuyendo al éxito escolar" (Lahire, 1995, p. 398). La autora continúa haciendo hincapié en que los padres esperan que sus hijos tengan una vida mejor que la de ellos, con mejores fuentes laborales, con trabajos menos mal pagados, "menos sucios" y más valorizado que el suyo.

En cualquier caso, la USB posee un alto porcentaje de estudiantes de primera generación (ver gráfico 3). Esta constatación representa un doble desafío institucional porque, por un lado, se debe mantener los costos de matrícula al nivel de posibilidades del presupuesto familiar y, por otro, se requiere mantener las existentes y promover iniciativas que ayuden a los estudiantes a alcanzar éxito académico de los destinatarios de los sectores populares. 
Gráfico 3: Estudiantes de primera generación.

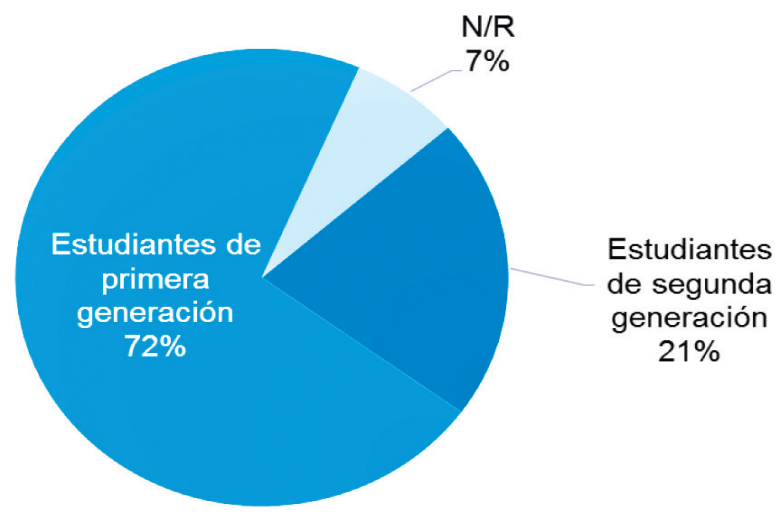

Fuente: Dirección de Planificación, USB. Estudio del Perfil del Estudiante de la USB 2018.

La propuesta educativa y pastoral de la Universidad Salesiana de Bolivia tiene un fuerte componente preferencial, la de los jóvenes de clases populares. El gráfico 3, es una aproximación a esa opción: el $72 \%$ de los estudiantes son de primera generación.

Asimismo, a este grupo de estudiantes de primera generación se les consultó acerca de la ocupación de la mamá y del papá. Las respuestas señalan que el 50\% de las madres de familia de estos estudiantes trabajan en ocupaciones relacionadas con lavado, aseo, servicios, ama de casa u otro similar. Por su parte, el $60 \%$ los papás de este grupo declara que se dedica a trabajos de obrero calificado, taxista, microempresario, vendedor, obrero no calificado, o similar.

Las preguntas que inmediatamente se hacen presentes nos deben llevar a reflexionar: ¿cómo mejorar los servicios educativo-pastorales de la USB para que estos estudiantes sientan un fuerte respaldo institucional para tener éxito en sus carreras universitarias? ¿Cómo involucrar a la familia para crear con ellos la corresponsabilidad de la educación de los jóvenes? Si existe una alta probabilidad que los estudiantes de primera generación reprueben asig- 
naturas o abandonen su carrera, es importante generar programas de retención orientados al apoyo académico de esta mayoría estudiantil.

\section{Procedencia geográfica de los estudiantes de la USB}

De acuerdo con el Informe de Desarrollo Humano 2016 del Programa de Nacionales Unidas para el Desarrollo (PNUD) en Bolivia, las ciudades se han convertido en el lugar de vivienda de dos terceras partes de la población total y se calcula que, hasta 2030, ocho de cada diez bolivianos vivirán en las ciudades, en particular en el eje central: La Paz, El Alto, Cochabamba y Santa Cruz de la Sierra (PNUD, 2016). Cabe mencionar que, junto al crecimiento demográfico de estas cuatro ciudades, se advierte un bono demográfico, que se puede considerar como un factor favorable para el desarrollo económico y social del país, porque gran parte de esta población estará entre los 18 y 40 años, que es considerado un periodo productivo.

Retomando la línea de análisis de la procedencia, la imagen 2 muestra los barrios de los cuales provienen los estudiantes de la Sede Central en La Paz. Administrativamente, la ciudad de La Paz está organizada en nueve macrodistritos, dos rurales y siete urbanos.

En lo concerniente a la distribución de la pobreza en el municipio..., del total de habitantes pobres, un $29 \%$ se encuentra en el macro distrito Max Paredes; en segundo lugar se encuentra el macro distrito Cotahuma con $19 \%$ y, finalmente, los macro distritos Periférica y San Antonio con $18 \%$ y 17\% respectivamente, lo que demuestra que las laderas representan mayores índices de pobreza. (Pardo, 2007, p. 4) 
Imagen 2: Macro distritos y principales barrios de los que provienen los estudiantes de La Paz.

\begin{tabular}{|c|c|c|}
\hline Macrodistrito & Principales barrios & $\%$ \\
\hline Periférica & $\begin{array}{l}\text { Achachicala } \cdot \text { Chuquiaguillo } \cdot \text { Villa Fátima } \cdot \text { Vino Tinto } \cdot 5 \text { Dedos } \cdot \text { Santiago de } \\
\text { Lacaya } \cdot \text { Rosasani }\end{array}$ & $32 \%$ \\
\hline Max Paredes & $\begin{array}{l}\text { Munaypata } \cdot \text { La Portada } \cdot \text { El Tejar } \cdot \text { Gran Poder } \cdot \text { Obispo Indaburo } \cdot \text { Chamoco } \\
\text { Chico } \cdot \text { Pura Pura } \cdot \text { Ciudadela Ferroviaria }\end{array}$ & $29 \%$ \\
\hline San Antonio & $\begin{array}{l}\text { San Antonio } \cdot \text { Villa Copacabana } \cdot \text { Pampahasi } \cdot \text { Valle Hermoso } \cdot \text { Kupini } \cdot \text { Villa } \\
\text { Armonía } \cdot \text { Callapa, } \cdot \text { San Isidro }\end{array}$ & $14 \%$ \\
\hline Cotahuma & $\begin{array}{l}\text { Sopocachi } \cdot \text { Alto Sopocachi } \cdot \text { Pasankeri } \cdot \text { Tembladerani } \cdot \text { Alpacoma } \cdot \text { Belén } • \\
\text { Tacagua } \text { San Pedro } \cdot \text { Bajo Llojeta }\end{array}$ & $12 \%$ \\
\hline Zona Sur & $\begin{array}{l}\text { Obrajes } \cdot \text { Bella Vista } \cdot \text { Bolonia } \cdot \text { Irpavi } \cdot \text { Calacoto } \cdot \text { Cota Cota } \cdot \text { Achumani } \cdot \text { Ovejuyo } \\
\cdot \text { Koani } \cdot \text { La Florida } \cdot \text { Següencoma } \cdot \text { San Miguel }\end{array}$ & $8 \%$ \\
\hline Zona Centro & $\begin{array}{l}\text { Casco Urbano Central } \cdot \text { San Jorge } \cdot \text { Miraflores } \cdot \text { Barrio Gráfico } \cdot \text { San Sebastián } \cdot \\
\text { Santa Bárbara } \cdot \text { Parque Urbano Central }\end{array}$ & $5 \%$ \\
\hline Mallasa & Amor de Dios $\cdot$ Mallasa $\cdot$ Muela del Diablo $\cdot$ Mallasilla $\cdot$ Jupapina & $0 \%$ \\
\hline \multicolumn{3}{|c|}{$\begin{array}{l}\checkmark 45 \% \text { de los estudiantes son } \\
\text { del Municipio de La Paz }\end{array}$} \\
\hline
\end{tabular}

Fuente: Dirección de Planificación, USB. Estudio del Perfil del Estudiante de la USB 2018.

De acuerdo con los datos de Pardo (2007), en la ciudad de La Paz los macrodistritos con mayores índices de pobreza son Max Paredes, Cotahuma, Periférica y San Antonio. Al contrastar estos datos de pobreza con los resultados de la imagen 2 , se evidencia que esos son los cuatro macrodistritos de los cuales proviene la gran mayoría de los estudiantes de la USB. Sumando la población estudiantil de estos cuatro macrodistritos, el resultado alcanza a un total de $87 \%$.

Por otra parte, la USB también recibe estudiantes que provienen de la ciudad de El Alto, ellos representan un 31\%. Esta ciudad, fundada en el año 1987, de acuerdo con datos del Instituto Nacional de Estadísticas (INE), tiene casi un millón de habitantes, de los cuales $83,4 \%$ son inmigrantes de otras ciudades y departamentos. "El Censo 2012 establece que 30\% de la población en El Alto es pobre, que equivale a 300.390 habitantes, que no cuentan con las condiciones apropiadas en servicios básicos, saneamiento, educación, acceso a la salud" (INE, 2018, p. 3). Adicionalmente, al menos el $60 \%$ menores de veinticinco años, lo que refleja una presencia mayoritaria de jóvenes. 
Imagen 3: Barrios de los que provienen los estudiantes de El Alto.

\begin{tabular}{|c|l|c|}
\hline Distrito & \multicolumn{1}{|c|}{ Principales barrios } & $\%$ \\
\hline Distrito 6 & Villa Ingavi, Ballivián, Alto Lima, 16 de Julio & $40 \%$ \\
\hline Distrito 1 & Villa Bolívar B, Villa Bolívar E, Villa Dolores, Villa & $28 \%$ \\
& Exaltación, Santiago I, Santiago II, Ciudad Satélite & $12 \%$ \\
\hline Distrito 3 & Cosmos 79, Villa Adela, Collpani, 10 de Mayo & $8 \%$ \\
\hline Distrito 4 & Río Seco, Mercedario & $7 \%$ \\
\hline Distrito 8 & Ventilla, Senkata & $4 \%$ \\
\hline Distrito 2 & Nuevos Horizontes, El Kenko & $1 \%$ \\
\hline Distrito 5 & Germán Busch & \\
\hline
\end{tabular}

Fuente: Dirección de Planificación, USB. Estudio del Perfil del Estudiante de la USB 2018.

Según Quispe (2009), “La población pobre de la ciudad de El Alto se concentra en mayor proporción en los distritos 3, 6, 2 y 1" (p. 66). De estos cuatros distritos más pobres proviene el $84 \%$ de los estudiantes alteños de la USB.

Un dato adicional que debe resaltarse es el porcentaje de jóvenes que tiene la ciudad de El Alto: 60\%. Esto constituye un bono demográfico de un potencial altamente favorable para el desarrollo económico y social de esa ciudad; sin embargo, este bono demográfico puede verse hondamente desperdiciado si estos jóvenes no adquieren las competencias y conocimientos necesarios para ser parte activa del desarrollo económico y social. Está demostrado que el capital humano es la principal fuente de desarrollo de las sociedades. Shultz, citado en Rama (2014), señala que "Toda actividad que contribuya a la formación para el trabajo es una inversión en capital humano, la cual constituye un sacrificio de recursos presentes con la intención de obtener un cantidad mayor de recursos y beneficios futuros" (Shultz en Rama, 2014, p. 32). El autor, evidentemente, hace un fuerte énfasis en la formación para el trabajo, que es importante para el desarrollo económico del país; sin embargo, lo que la USB propone, adicionalmente, es un espacio educativo y pastoral donde los estudiantes se forjen como 
"profesionales competentes, buenos cristianos y honestos ciudadanos", como reza parte de la misión de la USB.

Vale la pena adicionar que a este panorama de la procedencia geográfica se suma la naturaleza de la unidad educativa en la que los estudiantes terminaron el bachillerato. Por legislación, en Bolivia existen tres tipos unidades educativas, a saber: (a) unidades educativas fiscales, financiadas por el Tesoro General de la Nación; (b) unidades educativas privadas, financiadas por cuotas y matrículas de las familias, y (c) unidades educativas de convenios, las cuales son supervisadas por el Estado, respetando la administración por entidades sin fines de lucro, generalmente religiosas.

Gráfico 4: Naturaleza de la unidad educativa de bachillerato de los estudiantes de la USB.

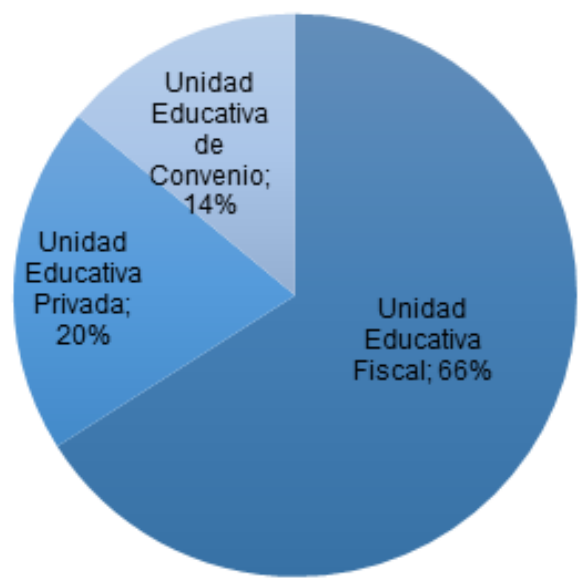

Fuente: Dirección de Planificación, USB. Estudio del Perfil del Estudiante de la USB 2018.

Si bien la asistencia a una unidad educativa no es proporcional a los ingresos familiares o clase social, existe una tendencia a que las familias de menos recursos económicos envíen a sus hijos a la educación fiscal.

\section{Reflexiones finales}

- Dada la cantidad de jóvenes que posee la población boliviana (un poco más de tres millones), es importante contar con pro- 
puestas educativas competitivas, acompañadas de políticas estatales al igual que el resto de la población. Es más que una voz que clama en el desierto, para transformarse en una demanda reivindicativa de estos jóvenes de sectores populares y pobres.

- En el ámbito político y económico, se ha generado una suerte de descontrol en el crecimiento del comercio informal. Con una juventud emprendedora, que carga una pesada mochila de las diferencias adversas, se sobrepone a estas circunstancias y no tiene miedo al cambio; por ello, los jóvenes creen en su formación, en el porvenir y en el crecimiento individual y social.

- La migración juvenil a las grandes ciudades, generada por la búsqueda de mejores oportunidades, no solo conlleva la desintegración familiar, sino que plantea al Estado una nueva problemática con variables hasta ahora desconocidas, que obliga a crear empleos y una mejor infraestructura social, y el desarrollo de los servicios educativos, en este caso, de formación profesional.

- La salida al encuentro de los jóvenes menos favorecidos obliga a los docentes a replantear la educación estandarizada, porque aparecen nuevas demandas que dependen de condiciones académicas y emocionales, que no encuentran el foco de referencia en casa para resolver sus problemas particulares y de rendimiento educativo.

- La procedencia geográfica de los estudiantes de la USB, tanto en la ciudad de La Paz como en la ciudad de El Alto, es una constatación del impacto de la misión de la USB para con los jóvenes menos favorecidos. Asimismo, la USB, por su ubicación geográfica, es una alternativa para los jóvenes de clases populares, dado que sus instalaciones a nivel nacional están ubicadas en ciudades intermedias o, en el caso de la Sede Central, en la periferia de la ciudad.

- La USB tiene un alto porcentaje de jóvenes que son considerados parte de la Generación Z. Estos jóvenes tienen un alto uso de las tecnologías de información y comunicación, especialmente de las redes sociales. A partir de la propuesta educativa- 
pastoral de la USB, es importante ayudarles a generar pensamiento crítico, que les permita ser profesionales competentes, buenos cristianos y honestos ciudadanos.

- Tener un alto número de estudiantes de primera generación conlleva responsabilidades de apoyo y acompañamiento a su desempeño académico y emocional. De no hacerse nada, existe una alta posibilidad de que los estudiantes tengan altos índices de reprobación, o, lo que es peor, queabandonen sus estudios universitarios, frustrando sus anhelos de conseguir una "mejor vida". Para acompañar a estos estudiantes, es fundamental abrir programas de retención y apoyo académico, que involucren actividades extracurriculares o cocurriculares.

- El desarrollo económico y social de una sociedad depende en gran medida del capital cultural de su población. Bolivia tiene y tendrá en los siguientes años un importante bono demográfico, el cual debe ser aprovechado por el país, brindando alternativas de profesionalización, especialmente para los menos favorecidos.

- Se puede afirmar, sin temor a equivocaciones, que la Universidad Salesiana de Bolivia cumple su misión de opción preferencial de brindar educación superior a los jóvenes menos favorecidos y de clases populares. Está demostrado que más del $80 \%$ de ellos viene de barrios con altos índices de pobreza, la ubicación geográfica demuestra una intencionalidad de ir en busca de nuestros destinatarios y, finalmente, la USB acoge a más del $70 \%$ de sus estudiantes de primera generación, cuyos padres, en su gran mayoría, trabajan en ocupaciones u oficios que son considerados menores o que no requieren un alto nivel de formación.

\section{Referencias bibliográficas}

Agencia de Gobierno Electrónico y Tecnologías de Información y Comunicación. (2018). Estado TIC: Estado de las Tecnologías de Información y Comunicación en el Estado Plurinacional de Bolivia. La Paz, Bolivia: Editorial del Estado Plurinacional de Bolivia 
Instituciones Salesianas de Educación Superior. (2016). Documento de Políticas para la Presencia Salesiana en la Educación Superior 2016-2021.

Instituto Nacional de Estadísticas de Bolivia. (2018). El Alto, la ciudad más joven de Bolivia. La Paz, Bolivia.

Lahire, B. (1995). Tableau de famille. Heurs et malheurs scolaire en milieux populaires. Paris: Le Seuil.

Ministerio de Autonomías de Bolivia. (2014). Agenda Patriótica del Bicentenario 2025. Recuperado de http://vpc.planificacion. gob.bo/uploads/recursos/AGENDA_PATRIOTICA2025_ QUIEN_HACE_QUE.pdf

Pardo, E. (2007). Jóvenes Aymaras, trabajadoras del hogar asalariadas en la Ciudad de La Paz. Cuaderno de Investigación, 1, La Paz, Bolivia.

Pérez de Rada, E. Demanda laboral en Bolivia. Conferencia divtada el 7 de marzo de 2018, Universidad Salesiana de Bolivia, La Paz.

Pew Research Center. (2018). Defining generations: Where Millennials end and post-Millennials begin. Recuperado de http:// www.pewresearch.org/fact-tank/2018/03/01/defininggenerations-where-millennials-end-and-post-millennials-begin/

Programa de las Naciones Unidas para el Desarrollo (PNUD). (2002). Informe de desarrollo humano 2002: PNUD ( $1^{\text {a }}$ ed.). La Paz, Bolivia: SPC Impresores S.A.

Quispe, G. M. (2009). La formación de la ciudad de El Alto y sus consecuencias (Tesis Doctoral). Recuperado de https://repositorio.uam.es/xmlui/bitstream/handle/10486/154/22637_quispe_fernandez_gabith_miriam. pdf? sequence $=1$ \&isAllowed $=y$

Rama, C. (2014). Los nuevos modelos universitarios en América Latina. San Salvador, El Salvador: Ediciones Universidad Evangélica de El Salvador.

Salesianos de Don Bosco. (2015). Constituciones y reglamentos. Madrid, España: Editorial CCS.

Soto, V. (2015). Estudiantes de primera generación en Chile: una aproximación cualitativa a la experiencia universitaria. Revista Complutense de Educación, 27(3), 1157-1173.

Universidad Salesiana de Bolivia. (2016). Modelo Académico de la USB. La Paz, Bolivia. 